\title{
Recurrent Sigmoid Volvulus Treated by Colonoscopic Reduction
}

\author{
Shinya Ito Akito Oshima Masahiko Inamori Takashi Uchiyama Hiroshi lida Hiroki Endo \\ Kunihiro Hosono Yasunari Sakamoto Koji Fujita Masato Yoneda Hirokazu Takahashi \\ Tomoko Koide Chikako Tokoro Ayumu Goto Yasunobu Abe Noritoshi Kobayashi \\ Kensuke Kubota Satoru Saito Atsushi Nakajima \\ Gastroenterology Division, Yokohama City University Hospital, Yokohama, Japan
}

Dear Sir,

A 49-year-old man was admitted to our hospital with a history of melena. The patient had a previous history of surgery for a left adrenal tumor and chronic constipation. Colonoscopy demonstrated a rectal ulcer with bleeding. Endoscopic hemostasis was successfully accomplished with a hemoclip. Conservative therapy was instituted following the procedure. On the 8 th hospital day, a liquid diet was started, because a follow-up colonoscopy revealed satisfactory healing of the ulcer. However, the patient did not pass stools even after the administration of laxatives. On the tenth day, he complained of abdominal pain. Abdominal X-ray showed dilated bowel loops with 'coffee bean' sign appearance (fig. 1) [1]. We made the diagnosis of sigmoid volvulus and attempted a colonoscopic reduction as the initial nonoperative treatment [2]. The reduction was successful, associated with a dramatic reduction of the symptoms.

Although the patient was started on parenteral nutrition and intravenous antibiotics after the reduction treatment, the volvulus recurred three times on the $12 \mathrm{th}$, 13th and 18th hospital days. Each recurrence was treated by colonoscopic reduction, and no necrotic tissues were observed endoscopically. After a series of recurrences, the symptoms, along with the inflam-

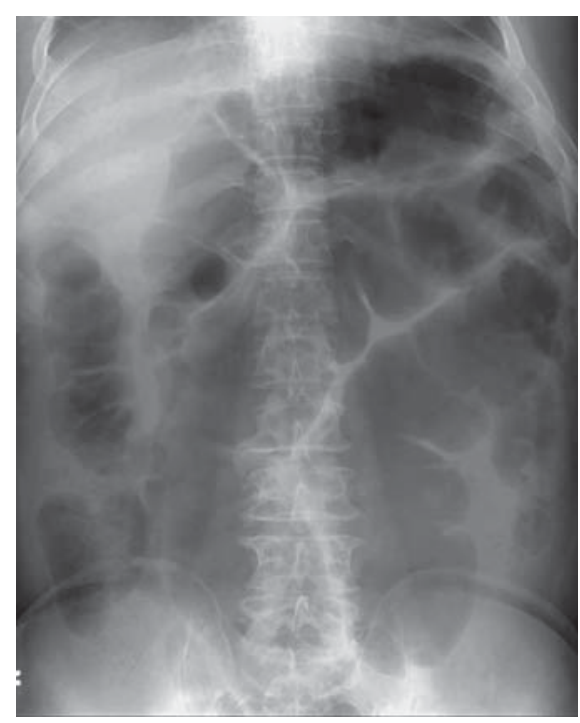

Fig. 1. Abdominal X-ray reveals the characteristic sign of sigmoid volvulus, described as the 'coffee bean' sign.

matory response, gradually subsided. The patient resumed a regular diet on the 24 th day, and was discharged on the 42 nd day. He has remained under regular review as an outpatient, but has not shown any signs of recurrence.
The risk factors implicated in sigmoid volvulus are chronic constipation, age, use of psychoactive drugs, and a history of abdominal surgery $[3,4]$. Our patient had constipation and a history of surgery. In addition, there are a few case reports of patients with the onset of sigmoid volvulus after colonoscopy.

In conclusion, we encountered a patient who developed sigmoid volvulus after colonoscopy. Our experience suggests that colonoscopy could be a potential risk factor for the development of sigmoid volvulus.

\section{References}

1 Atamanalp SS, Yildirgan MI, Başoğlu M, Oren D, Aydinli B, Kargi A, Onbaş O: Retraction. Clinical presentation and diagnosis of sigmoid volvulus: outcomes of 40 -year and 859-patient experience. J Gastroenterol Hepatol 2009;24:1154.

2 Anderson JR, Lee D: The management of acute sigmoid volvulus. Br J Surg 1981;68: 117.

-3 Friedman JD, Odland MD, Bubrick MP: Experience with colonic volvulus. Dis Colon Rectum 1989;32:409.

4 Alshawi JS: Recurrent sigmoid volvulus in pregnancy: report of a case and review of the literature. Dis Colon Rectum 2005;48:1811.

\section{KARGER}

Fax +41613061234 E-Mail karger@karger.ch www.karger.com
M. Inamori, $\mathrm{MD}, \mathrm{PhD}$

Gastroenterology Division, Yokohama City University Hospital

3-9 Fukuura, Kanazawa-ku

Yokohama, 236-0004 (Japan)

Tel. +81 45787 2640, Fax +81 45784 3546, E-Mail inamorim@med.yokohama-cu.ac.jp 